\author{
Hitoshi Sakuraba $\cdot$ Mai Murata-Ohsawa \\ Ikuo Kawashima $\cdot$ Youichi Tajima $\cdot$ Masaharu Kotani \\ Toshio Ohshima $\cdot$ Yasunori Chiba $\cdot$ Minako Takashiba \\ Yoshifumi Jigami · Tomoko Fukushige \\ Tamotsu Kanzaki $\cdot$ Kohji Itoh

\section{Comparison of the effects of agalsidase alfa and agalsidase beta on cultured human Fabry fibroblasts and Fabry mice}

Received: 6 September 2005/ Accepted: 3 November 2005/Published online: 22 December 2005

(c) The Japan Society of Human Genetics and Springer-Verlag 2005

\begin{abstract}
We compared two recombinant $\alpha$-galactosidases developed for enzyme replacement therapy for Fabry disease, agalsidase alfa and agalsidase beta, as to specific $\alpha$-galactosidase activity, stability in plasma, mannose 6phosphate (M6P) residue content, and effects on cultured human Fabry fibroblasts and Fabry mice. The specific enzyme activities of agalsidase alfa and agalsidase beta were 1.70 and $3.24 \mathrm{mmol} \mathrm{h}^{-1} \mathrm{mg}$ protein $^{-1}$, respectively, and there was no difference in stability in plasma between them. The M6P content of agalsidase beta (3.6 $\mathrm{mol} / \mathrm{mol}$ protein) was higher than that of agalsidase
\end{abstract}

H. Sakuraba $(\bowtie) \cdot M$. Murata-Ohsawa · I. Kawashima

Y. Tajima $\cdot$ M. Kotani

Department of Clinical Genetics,

The Tokyo Metropolitan Institute of Medical Science,

Tokyo Metropolitan Organization for Medical Research,

3-18-22 Honkomagome, Bunkyo-ku,

Tokyo 113-8613, Japan

E-mail: sakuraba@rinshoken.or.jp

Tel.: +81-3-38232105

Fax: $+81-3-38236008$

M. Murata-Ohsawa

CREST, JST, Kawaguchi, Japan

T. Ohshima

Laboratory for Developmental Neurology,

Brain Science Institute, RIKEN, Wako, Japan

Y. Chiba $\cdot$ M. Takashiba $\cdot$ Y. Jigami

Research Center for Glycoscience,

National Institute of Advanced Industrial Science and Technology,

Tsukuba, Japan

T. Fukushige $\cdot$ T. Kanzaki

Department of Dermatology,

Kagoshima University Graduate School of Medical and

Dental Sciences, Kagoshima, Japan

K. Itoh

Department of Medicinal Biotechnology,

Institute for Medicinal Resources,

Graduate School of Pharmaceutical Sciences,

The University of Tokushima, Tokushima, Japan alfa $(1.3 \mathrm{~mol} / \mathrm{mol}$ protein $)$. The administration of both enzymes resulted in marked increases in $\alpha$-galactosidase activity in cultured human Fabry fibroblasts, and Fabry mouse kidneys, heart, spleen and liver. However, the increase in enzyme activity in cultured fibroblasts, kidneys, heart and spleen was higher when agalsidase beta was used. An immunocytochemical analysis revealed that the incorporated recombinant enzyme degraded the globotriaosyl ceramide accumulated in cultured Fabry fibroblasts in a dose-dependent manner, with the effect being maintained for at least 7 days. Repeated administration of agalsidase beta apparently decreased the number of accumulated lamellar inclusion bodies in renal tubular cells of Fabry mice.

Keywords Fabry disease $\alpha$-Galactosidase · Globotriaosyl ceramide · Enzyme replacement therapy $\cdot$ Fabry mouse

\section{Introduction}

Fabry disease (MIM 301500) is an X-linked genetic disease arising from a deficiency of $\alpha$-galactosidase (EC 3.2.1.22) activity (Desnick et al. 2001). The enzyme cleaves terminal $\alpha$-D-galactosyl residues from glycolipids, primarily globotriaosyl ceramide (GL-3), in lysosomes. The enzyme defect results in lysosomal accumulation of GL-3, which is recognized as characteristic lamellar inclusion bodies by electron microscopy. Male patients with classic Fabry disease develop pain in the peripheral extremities, hypohidrosis, angiokeratoma, renal disorders, and cardiovascular and cerebrovascular involvement. The incidence of classic Fabry disease has been estimated to be 1 in 40,000 male newborns. Patients with variant form Fabry disease have milder clinical manifestations, which are sometimes limited to the heart. Although the incidence of the variant form of Fabry disease 
has not been determined yet, 7 out of 230 Japanese males with left ventricular hypertrophy were found to have variant Fabry disease (Nakao et al. 1995). Furthermore, a recent survey has revealed that many Fabry heterozygous females can be affected similarly to hemizygous males (Mehta et al. 2004). Taking this into account, the number of Fabry patients requiring treatment is now thought to be much larger than previously expected.

Two different human recombinant $\alpha$-galactosidases have been developed for enzyme replacement therapy for Fabry disease (Desnick et al. 2003). One is agalsidase beta (trade name Fabrazyme; Genzyme Therapeutics, Cambridge, MA) produced in Chinese hamster ovary (CHO) cells (Eng et al. 2001b). Agalsidase beta has been approved in Europe, the United States, and Japan, and, so far, approximately 1,200 Fabry patients have been treated with this drug. The other is agalsidase alfa (trade name Replagal; Transkaryotic Therapies, Cambridge, MA) generated in human fibroblasts (Schiffmann et al. 2000). Agalsidase alfa has been approved in Europe, and about 500 Fabry patients have been treated with this drug. In Japan, a clinical trial of agalsidase alfa has been completed and it is under evaluation for approval by the Ministry of Health, Labor and Welfare of Japan, and patients who were involved in the clinical trial have continued to be treated with agalsidase alfa with the permission of the Ministry.

In view of this background, strong requests have been made by physicians involved in the treatment of this disease for investigation of the differences in biochemical characteristics and effects between these two recombinant enzymes. However, little information is available because different endpoints and dosing regimens were used in the clinical trials of these drugs. Lee et al. (2003) reported the biochemical and pharmacological examination of these two preparations. However, considering that these authors are employed by Genzyme, requests have been made for comparison of the effects of these drugs by a third party that is not affiliated with either of the manufacturers.

For this study, we obtained agalsidase alfa and agalsidase beta for administration to Fabry patients in Japan and compared them in terms of specific enzyme activity, stability in plasma, sugar chain monosaccharide composition, and the increase in $\alpha$-galactosidase activity in cultured human Fabry fibroblasts and organs of $\mathrm{Fa}-$ bry mice upon administration of agalsidase alfa and agalsidase beta. Furthermore, we examined the effect of agalsidase beta on cleavage of the accumulated GL-3 in cultured Fabry fibroblasts and kidney tissues of Fabry mice.

\section{Materials and methods}

Agalsidase alfa and agalsidase beta

Agalsidase alfa, as a liquid formulation with a concentration of $1 \mathrm{mg} / \mathrm{ml}$, was provided by Sumitomo Phar- maceuticals (Osaka, Japan). Agalsidase beta, as a lyophilized powder, was purchased from Genzyme Japan (Tokyo, Japan), and was reconstituted according to the manufacturer's instructions to a concentration of $5 \mathrm{mg} /$ $\mathrm{ml}$, and then diluted to $1 \mathrm{mg} / \mathrm{ml}$ before use. The $\alpha$-galactosidase activity of these enzyme preparations was determined immediately after we obtained them.

$\alpha$-Galactosidase assay

$\alpha$-Galactosidase activity was measured fluorometrically with 4-methylumbelliferyl- $\alpha$-D-galactopyranoside (Calbiochem, San Diego, CA) as a substrate and $N$-acetyl-Dgalactosamine (Sigma, St. Louis, MO) as an inhibitor for $\alpha$ - $N$-acetylgalactosaminidase according to the method previously reported (Mayes et al. 1981). Protein determination was performed with a DC assay kit (BioRad, Richmond, CA), using bovine serum albumin (BSA) as a standard.

Stability of agalsidase alfa and agalsidase beta in plasma

Agalsidase alfa and agalsidase beta were separately added to human plasma to give $1.6-1.7 \mu \mathrm{mol} \mathrm{h}^{-1} \mathrm{ml}^{-1}$ activity, and the plasma was then kept at $37^{\circ} \mathrm{C}$. Subsequently, $\alpha$-galactosidase activity in the plasma was measured at $10 \mathrm{~min}$ intervals until $120 \mathrm{~min}$ after enzyme addition. Taking the $\alpha$-galactosidase activity in the plasma at time zero as 100 , the stability of agalsidase alfa and agalsidase beta in the plasma was expressed as the ratio $(\%)$ of $\alpha$-galactosidase activity at each time point to the time zero value.

\section{Monosaccharide analysis}

Agalsidase alfa and agalsidase beta were hydrolyzed with $2 \mathrm{M}$ trifluoroacetic acid and L-rhamnose as an internal standard at $100^{\circ} \mathrm{C}$ for $2 \mathrm{~h}$, and monosaccharides derived from the sugar chains were analyzed by means of capillary electrophoresis using a P/ACE MDQ equipped with a laser-induced fluorescence detector (Beckman Coulter, Fullerton, CA) according to the method previously reported (Takashiba et al. 2004). Authentic monosaccharides were used as standards for quantitation. The results were compared with those previously reported (Lee et al. 2003).

$\alpha$-Galactosidase activity increase in cultured human Fabry fibroblasts on administration of agalsidase alfa and agalsidase beta

Cultured fibroblasts from a patient with Fabry disease and a normal control subject were established and maintained in our laboratory. The cells were cultured in 
Ham's F-10 medium containing 10\% fetal calf serum and antibiotics at $37^{\circ} \mathrm{C}$ in a humidified incubator flushed continuously with a $5 \% \mathrm{CO}_{2}-95 \%$ air mixture. The study involving the cultured fibroblasts was approved by the Ethical Committee of our institute.

Agalsidase alfa and agalsidase beta were adjusted and added to the culture medium of Fabry fibroblasts to a concentration of $1 \mu \mathrm{g} / \mathrm{ml}$ or to give an enzyme activity of 1.6-1.7 $\mu \mathrm{mol} \mathrm{h}^{-1} \mathrm{ml}^{-1}$. After 3 days incubation, the cells were harvested mechanically, washed three times with phosphate-buffered saline (PBS), $\mathrm{pH} 7.4$, and then collected as a pellet by centrifugation. An appropriate amount of water was then added to the pellet, the cells disrupted with ultrasonic waves, and the cell-associated $\alpha$-galactosidase activity in the cell homogenate was determined.

$\alpha$-Galactosidase activity increase in organs of Fabry mice upon administration of agalsidase alfa and agalsidase beta

Wild type C57BL/6 mice (wild type group) and Fabry mice ( $\alpha$-galactosidase knock-out mice, donated by A.B. Kulkarni; Ohshima et al. 1997, 1999) were used in this experiment according to the rules drawn up by the Animal Care Committee of our institute. Fabry mice were divided into five groups, i.e., an untreated Fabry group (Fabry/untreated group), a Fabry group administered agalsidase alfa and another administered agalsidase beta at $0.5 \mathrm{mg} / \mathrm{kg}$ body weight (Fabry/agalsidase-alfa $/ 0.5$ group and Fabry/agalsidase-beta/0.5 group, respectively), and a Fabry group administered agalsidase alfa and another administered agalsidase beta at $3.0 \mathrm{mg} / \mathrm{kg}$ body weight (Fabry/agalsidase-alfa/3.0 group and Fabry/agalsidase-beta/3.0 group, respectively). Each group consisted of three mice. The indicated dose of the enzyme was injected into the tail veins of mice belonging to the Fabry/agalsidase-alfa/0.5, Fabry/agalsidase-beta/0.5, Fabry/agalsidase-alfa/3.0, and Fabry/agalsidase-beta/3.0 groups. The mice in each group were sacrificed at $2 \mathrm{~h}$ after administration of the enzyme, and their kidneys, hearts, spleens and livers were collected. Tissue samples were then homogenized in citrate-phosphate buffer, $\mathrm{pH} 4.6$, centrifuged, and the supernatants assayed for $\alpha$-galactosidase activity.

Examination of the effect of agalsidase beta on degradation of GL-3 accumulated in cultured Fabry fibroblasts

Fabry fibroblasts were cultured with culture medium containing $3.0 \mu \mathrm{g} / \mathrm{ml}$ agalsidase beta for $0,1,3,5$, and 7 days. Alternatively, fibroblasts were cultured with culture medium containing agalsidase beta at a concentration of $0,0.5,1.0,2.0$, or $3.0 \mu \mathrm{g} / \mathrm{ml}$ for 3 days. Following culture, immunocytochemical analysis of GL3 and $\alpha$-galactosidase was performed. Cells grown on a chamber slide (Nunc, Naperville, IL) were fixed with $2 \%$ paraformaldehyde in PBS, pH 7.4 for $10 \mathrm{~min}$, followed by blocking with $5 \%$ BSA in PBS for $1 \mathrm{~h}$. The cells were then reacted with a mouse monoclonal antibody to GL3 (IgG isotype, Kotani et al. 1994) and rabbit polyclonal antibodies to $\alpha$-galactosidase (diluted 1:100; IgG isotype, Ishii et al. 1994) for $1 \mathrm{~h}$. After washing, the cells were further reacted for $1 \mathrm{~h}$ with a fluorescent isothiocyanateconjugated goat anti-mouse $\operatorname{IgG~} \mathrm{F}\left(\mathrm{ab}^{\prime}\right)_{2}$ (diluted 1:200; Jackson Immuno Research, West Grove, PA) and a rhodamine-conjugated goat anti-rabbit $\operatorname{IgG~} \mathrm{F}\left(\mathrm{ab}^{\prime}\right)_{2}$ (diluted 1:400; Jackson Immuno Research). The stained cells were observed under a microscope (Axiovert $100 \mathrm{M}$; Carl Zeiss, Oberkochen, Germany) equipped with a confocal laser scanning imaging system (LSM510; Carl Zeiss).

\section{Electron microscopy}

A morphological study was performed to examine the incorporation of the enzyme into kidney tissues in this study, because kidneys are one of the organs affected in Fabry disease and a previous study revealed that enzyme uptake by kidney tissues was not high (Thunberg et al. 2002). Three milligrams of agalsidase beta $/ \mathrm{kg}$ body weight was injected into Fabry mice every week for 4 weeks, and the mice were killed 6 days after the last injection. Kidney tissues from untreated and treated Fabry mice were collected, cut into small pieces, and fixed in cold $2.5 \%$ glutaraldehyde and $2 \%$ paraformaldehyde in PBS. The specimens were rinsed in PBS overnight, and then postfixed with $2 \%$ osmium tetraoxide in $0.2 \mathrm{M}$ sucrose in PBS for $1 \mathrm{~h}$, and dehydrated with ethanol and glycidyl $n$-butylether. After dehydration, the specimens were embedded in Epon. Sections $(0.1 \mu \mathrm{m}$ thick) were then prepared, stained with $2 \%$ uranyl acetate in 50\% ethanol, and counterstained with Reynold lead citrate for $3 \mathrm{~min}$, and finally examined under an electron microscope (Hitachi H-7100; Hitachi, Tokyo).

\section{Results}

Specific enzyme activity of agalsidase alfa and agalsidase beta

The specific enzyme activities of agalsidase alfa and agalsidase beta determined by the fluorescent method using 4-methylumbelliferyl- $\alpha$-D-galactopyranoside as substrate were 1.70 and $3.24 \mathrm{mmol} \mathrm{h}^{-1} \mathrm{mg}_{\text {protein }}^{-1}$, respectively.

Stability of agalsidase alfa and agalsidase beta in plasma

A time course of changes in enzyme activity of agalsidase alfa and agalsidase beta in plasma at $37^{\circ} \mathrm{C}$ revealed 


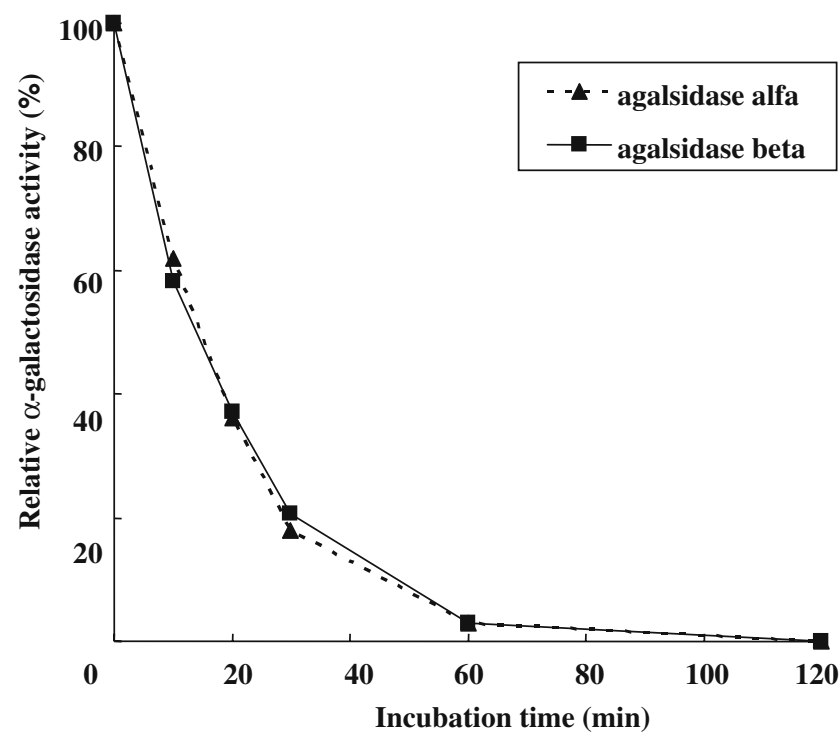

Fig. 1 Time course of $\alpha$-galactosidase activity of agalsidase alfa and agalsidase beta in plasma at $37^{\circ} \mathrm{C}$

that there was no difference in stability between agalsidase alfa and agalsidase beta under these conditions (Fig. 1).

Monosaccharide composition of the sugar chains of agalsidase alfa and agalsidase beta

The monosaccharides in the sugar chains of agalsidase alfa and agalsidase beta, especially their mannose 6-phosphate (M6P) residue content, were analyzed and

Table 1 Monosaccharide analysis. M6P Mannose-6-phosphate, GlcNAc $N$-acetylglucosamine

\begin{tabular}{lll}
\hline & $\begin{array}{l}\text { Our study } \\
(\mathrm{mol} / \mathrm{mol} \text { protein })\end{array}$ & $\begin{array}{l}\text { Lee et al. 2003 } \\
\text { (mol/mol protein) }\end{array}$ \\
\hline $\begin{array}{ll}\text { Agalsidase alfa } \\
\text { M6P }\end{array}$ & $1.3 \pm 0.1$ & \\
Galactose & $9.1 \pm 1.9$ & $1.8 \pm 0.0$ \\
Fucose & $4.7 \pm 0.1$ & $12.2 \pm 1.0$ \\
Mannose & $27.6 \pm 0.1$ & $3.0 \pm 0.3$ \\
GlcNAc & $7.3 \pm 2.2^{\mathrm{b}}$ & $27.6 \pm 0.5$ \\
Sialic acid & $\mathrm{ND}^{\mathrm{c}}$ & $22.5 \pm 2.3$ \\
Agalsidase beta & & $6.9 \pm 0.6$ \\
M6P & $3.6 \pm 0.3$ & \\
Galactose & $6.4 \pm 0.2$ & $3.1 \pm 0.1$ \\
Fucose & $2.1 \pm 0.1$ & $1.0 \pm 0.4$ \\
Mannose & $25.7 \pm 0.9$ & $25.7 \pm 1.8$ \\
GlcNAc & $7.9 \pm 0.1^{\mathrm{b}}$ & $18.4 \pm 0.4$ \\
Sialic acid & $\mathrm{ND}^{\mathrm{c}}$ & $7.0 \pm 1.0$ \\
\end{tabular}

${ }^{\text {a }}$ Values are expressed as mean $\pm \mathrm{SD}, n=3$ (agalsidase alfa) and 6 (agalsidase beta)

${ }^{b}$ Hydrolysis of GlcNAc residues bound to asparagine residues is probably not complete under the conditions employed in this experiment

${ }^{\mathrm{c}}$ Not determined compared with previously reported values (Lee et al. 2003). The M6P content was apparently higher in agalsidase beta than in agalsidase alfa (Table 1). The values determined for monosaccharides, including M6P, galactose, fucose, and mannose, were almost the same as those reported by Lee et al. (2003) except for the value for $\mathrm{N}$-acetylglucosamine (GlcNAc). The difference is probably due to the fact that GlcNAc binds strongly to asparagine and hydrolysis of GlcNAc residues bound to asparagine residues was not complete under the conditions employed here. Unfortunately, the amount of agalsidase alfa donated was small and so we could not reexamine this. We did not determine the sialic acid content.

Increase in $\alpha$-galactosidase activity in cultured human Fabry fibroblasts upon administration of agalsidase alfa and agalsidase beta

Agalsidase alfa and agalsidase beta were separately added to the culture medium of fibroblasts derived from a patient with Fabry disease so that the final concentration of the protein or the enzyme activity was the same. After incubation in this medium for 3 days, the cells were harvested and the cell-associated $\alpha$-galactosidase activity determined. The $\alpha$-galactosidase activity in untreated Fabry fibroblasts was almost nil, and cell-associated $\alpha$-galactosidase activity apparently increased when agalsidase alfa or agalsidase beta was added to the culture medium (Table 2). The increase in cell-associated $\alpha$-galactosidase activity was always higher in the presence of agalsidase beta, regardless of whether enzyme protein concentration or enzyme activity was maintained constant.

Increase in $\alpha$-galactosidase activity in organs of Fabry mice upon administration of agalsidase alfa and agalsidase beta

Following injection of agalsidase alfa and agalsidase beta into the blood vessels of Fabry mice, the increases in $\alpha$-galactosidase activity in the kidneys, heart, spleen and liver were compared between the different groups. The $\alpha$-galactosidase activity in the organs of untreated Fabry mice was markedly lower than that in those of wild type mice (Fig. 2). The $\alpha$-galactosidase activity in each organ of Fabry mice increased in a dose-dependent manner upon intravascular administration of agalsidase alfa and agalsidase beta. After injection of agalsidase alfa and agalsidase beta at $0.5 \mathrm{mg} / \mathrm{kg}$ body weight, the enzyme activity in the kidneys, heart, spleen and liver increased by about 40-70, 80-120, 30-60, and 300$340 \%$, respectively, compared to levels in wild type mice. Administration of the enzymes at $3.0 \mathrm{mg} / \mathrm{kg}$ body weight markedly increased the enzyme activity in these organs to several times the levels in wild type mice. 
Table 2 Effects of agalsidase alfa and agalsidase beta on cultured Fabry fibroblasts

\begin{tabular}{lllll}
\hline & $\begin{array}{l}\text { Substance added to } \\
\text { the culture medium }\end{array}$ & \multicolumn{2}{l}{$\begin{array}{l}\text { Final concentration } \\
(\mu \mathrm{g} / \mathrm{ml})\end{array}$} & $\begin{array}{l}\text { Cell-associated } \alpha \text {-galactosidase } \\
\text { activity }\left(\mathrm{nmol} \mathrm{h}^{-1} \mathrm{mg}^{-1}\right)\end{array}$ \\
\hline Normal fibroblasts & - & - & - & 57 \\
Fabry fibroblasts & - & - & 1.70 & 1 \\
& Agalsidase alfa & 1 & 1.62 & $3.80 \times 10^{3}$ \\
& Agalsidase beta & 0.5 & 3.24 & $4.61 \times 10^{3}$ \\
& Agalsidase beta & 1 & $8.40 \times 10^{3}$ \\
\hline
\end{tabular}

When the effects of agalsidase alfa and agalsidase beta were compared, the increase in $\alpha$-galactosidase activity in the kidneys, heart and spleen was always greater after agalsidase beta administration than after agalsidase alfa administration. However, hardly any difference was observed in the liver.

\section{Kidney}

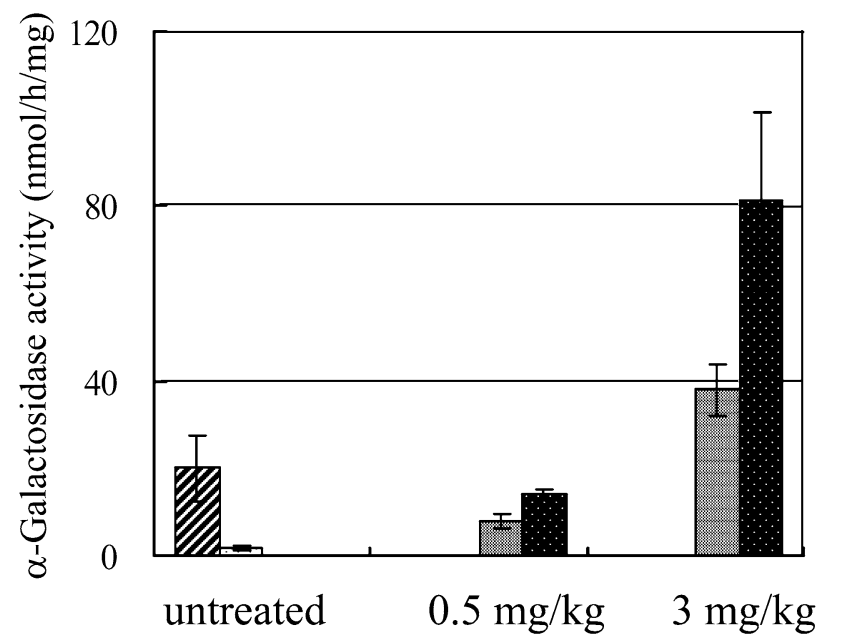

Spleen

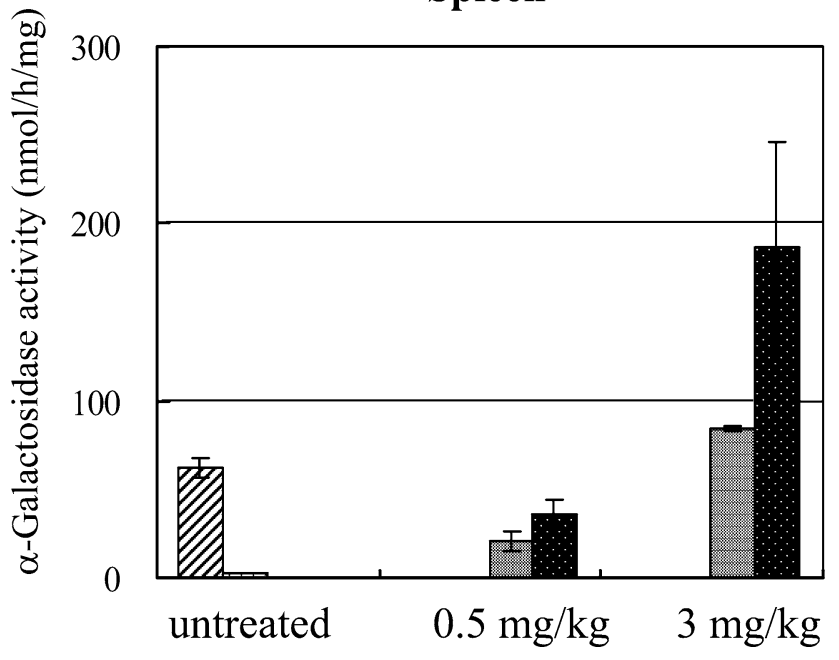

Fig. 2 Effects of agalsidase alfa and agalsidase beta on Fabry mice. Two doses of agalsidase alfa or agalsidase beta, i.e., 0.5 and $3.0 \mathrm{mg} / \mathrm{kg}$ body weight, were separately injected into the blood vessels of Fabry mice. The mice were sacrificed $2 \mathrm{~h}$ after injection,
Degradation of GL-3 accumulated in cultured Fabry fibroblasts upon administration of agalsidase beta

We examined incorporation of agalsidase beta into cultured Fabry fibroblasts, and its effect on degradation of GL-3 accumulated in the cells. As the amount of agalsi-

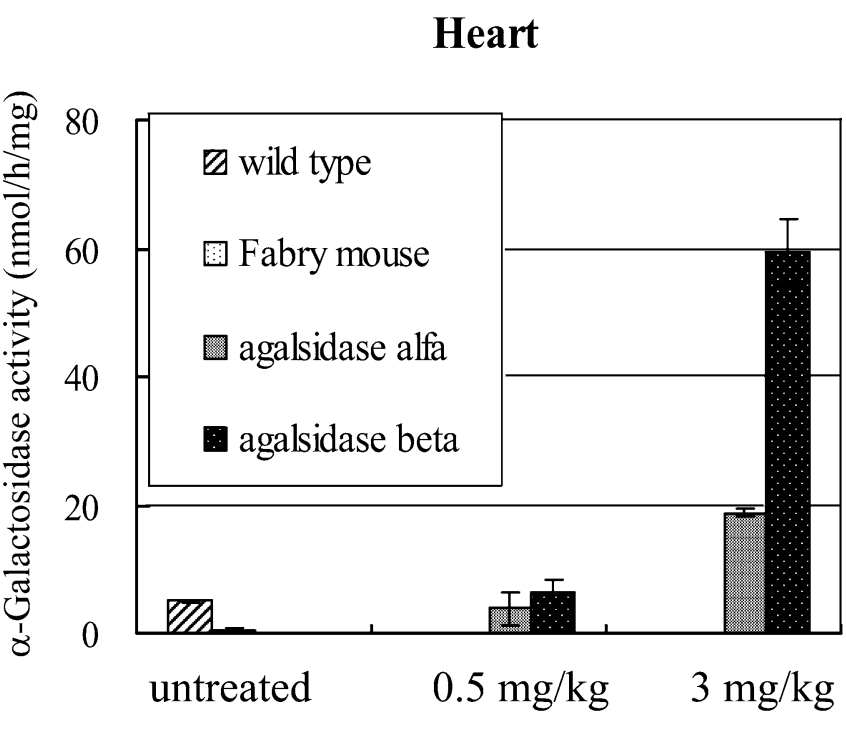

Liver

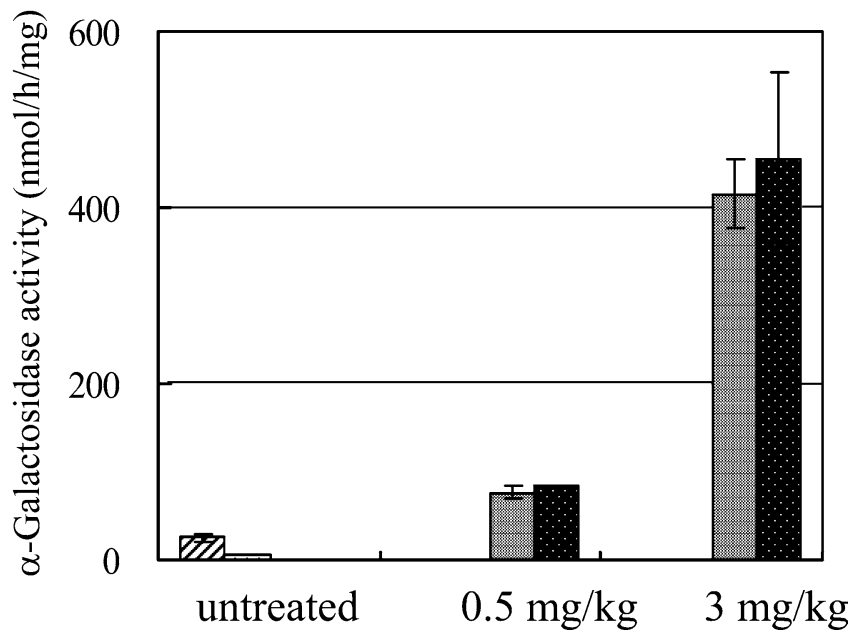

and $\alpha$-galactosidase activity in the kidneys, heart, spleen and liver was determined. Enzyme activities in untreated wild type and Fabry mice were also determined as controls. Values are expressed as means. Bars Standard deviation 
a
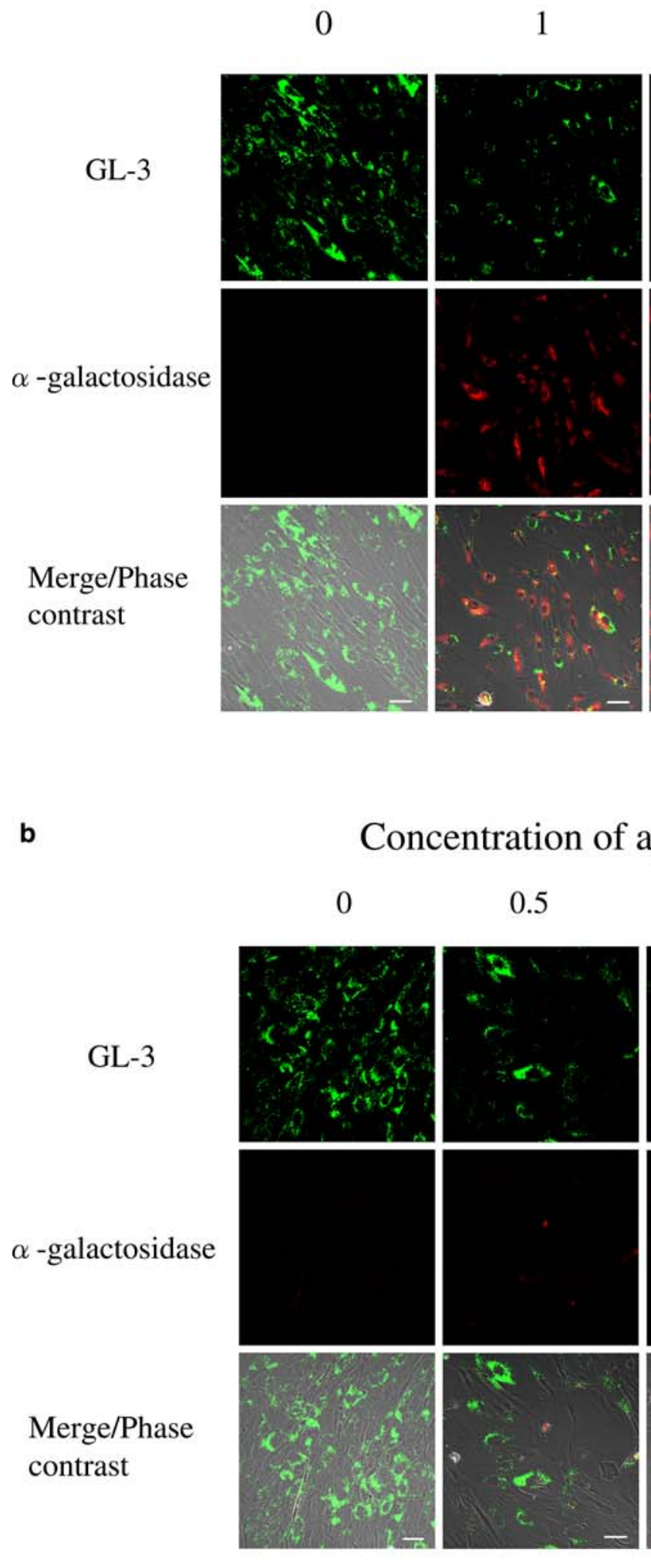
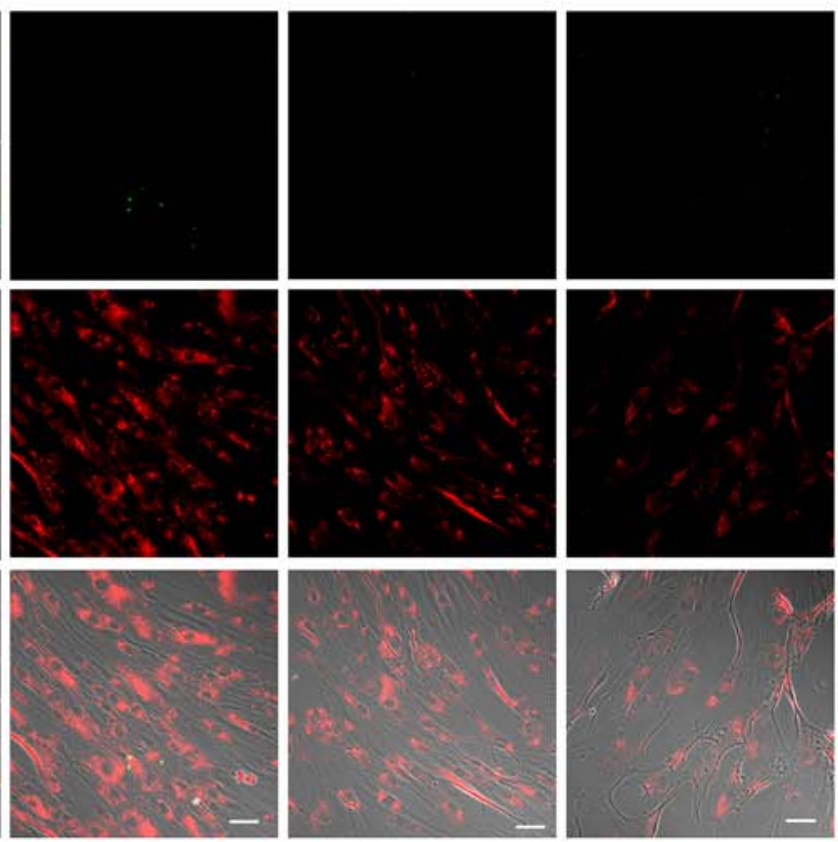

Bars, $50 \mu \mathrm{m}$

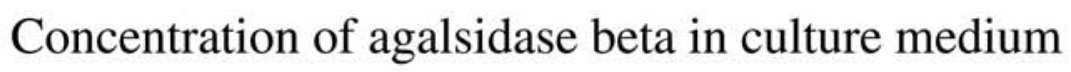

1.0
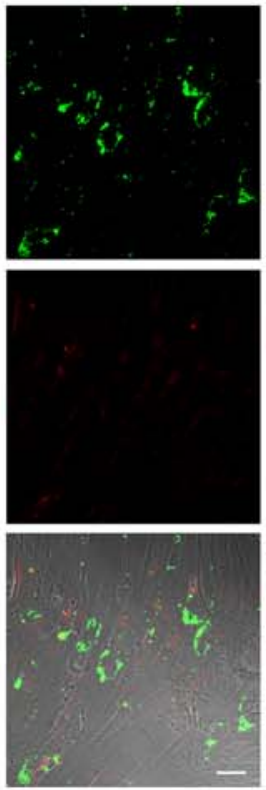

2.0
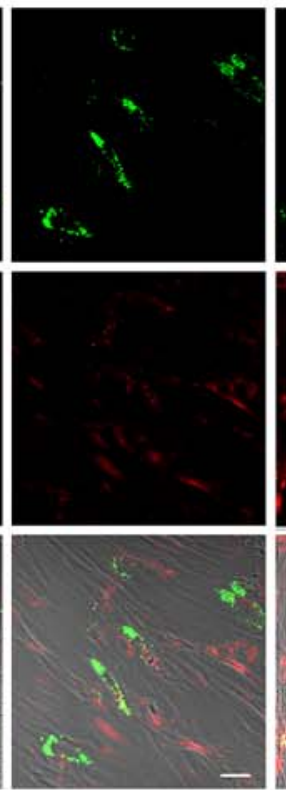

$\mu \mathrm{g} / \mathrm{ml}$
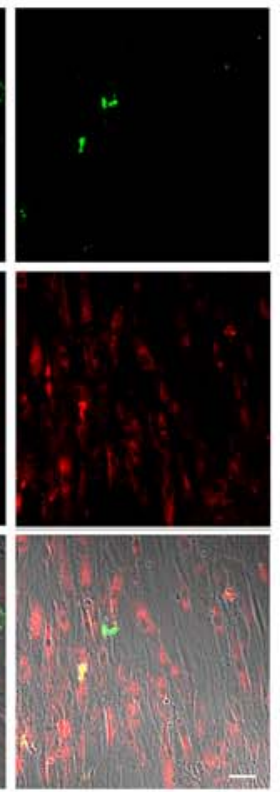

Bars, $50 \mu \mathrm{m}$

Fig. 3a,b Immunostaining for GL-3 and $\alpha$-galactosidase in Fabry fibroblasts after addition of agalsidase beta. Upper panels GL-3 stained with a monoclonal antibody to GL-3 (green), middle panels $\alpha$-galactosidase, stained with polyclonal antibodies to $\alpha$-galactosidase (red); lower panels merge/phase contrast, overlapped images with the two fluorescent probes and phasecontrast images. a Time-course, b dose-dependency. Bars $50 \mu \mathrm{m}$ 
dase alfa supplied was small, we could not compare its effect with that of agalsidase beta. The result of the timecourse analysis is shown in Fig. 3a. In untreated Fabry fibroblasts, strong immunofluorescence of GL-3 was observed, although no immunofluorescence of $\alpha$-galactosidase could be detected. In this experiment, agalsidase beta was added to the culture medium at a concentration of $3 \mu \mathrm{g} / \mathrm{ml}$. After 1 day of culture, $\alpha$-galactosidase immunofluorescence could be detected and that of GL-3 was apparently decreased. Maximum $\alpha$-galactosidase immunofluorescence was observed after 3 days of culture, with immunofluorescence gradually decreasing with time thereafter. Cleavage of the accumulated GL-3 was maintained for at least for 7 days after the addition of agalsidase beta. The result of the dose-dependency analysis is shown in Fig. 3b. Fabry fibroblasts were cultured for 3 days with culture medium containing agalsidase beta at various concentrations. Double staining for $\alpha$ galactosidase and GL-3 was then performed. Degradation of GL-3 in response to incorporation of agalsidase beta occurred in a dose-dependent manner.

Cleavage of lamellar inclusion bodies in renal tubular cells upon repeated administration of agalsidase beta

Agalsidase beta was repeatedly injected into Fabry mice, and their kidneys were then analyzed morphologically. Many lamellar inclusion bodies were observed in renal tubular cells of untreated Fabry mice (Fig. 4a), and their number markedly decreased with repeated administration of agalsidase beta (Fig. 4b).

\section{Discussion}

The phase 3 clinical trial of agalsidase alfa evaluated its effect in terms of reduction of Fabry-associated pain in the peripheral extremities, and a dose of $0.2 \mathrm{mg}$ agalsi- dase alfa $/ \mathrm{kg}$ body weight every 2 weeks was injected (Schiffmann et al. 2000). On the other hand, in the phase $1 / 2$ clinical trial of agalsidase beta, five doses of the enzymes, i.e., $0.3,1.0$, and $3.0 \mathrm{mg} / \mathrm{kg}$ body weight biweekly, and 1.0 and $3.0 \mathrm{mg} / \mathrm{kg}$ body weight every 2 days, were separately injected (Eng et al. 2001a). The phase 3 clinical trial evaluated the histological clearance of accumulated GL-3 in microvascular endothelial cells, and a dose of $1.0 \mathrm{mg}$ agalsidase beta $/ \mathrm{kg}$ body weight every 2 weeks was chosen (Eng et al. 2001b). Thus, the differences in the endpoint and doses of the drugs make it difficult to compare the effects of agalsidase alfa and agalsidase beta. In animal experiments, four doses of agalsidase beta, i.e., $0.3,1.0,3.0$, and $10.0 \mathrm{mg} / \mathrm{kg}$ body weight, were injected every 2 days into Fabry mice (Ioannou et al. 2001).

In this study, we examined the biochemical characteristics and effects of these drugs using preparations for Fabry patients in Japan as samples. When the $\alpha$-galactosidase activities of agalsidase alfa and agalsidase beta were compared, that of agalsidase beta was higher than that of agalsidase alfa. According to the report by Lee et al. (2003), who used agalsidase alfa and agalsidase beta samples available in Europe in their comparative study, there was not much difference between them. Since the enzyme activity of agalsidase beta reported by Lee et al. (2003) was almost the same as that determined by us, the enzyme activity of the agalsidase alfa preparation obtained in this study was probably decreased to some extent, although the reason for this has not yet been determined. As both enzyme proteins are derived from human $\alpha$-galactosidase cDNA, and the protein sequence is almost the same for both products, differences in formulation might have affected their stability. As to the stability in plasma, there was no difference between the two enzymes under the incubation conditions used in this study.

For enzyme replacement therapy for Fabry disease, targeting of the enzyme to affected organs is very

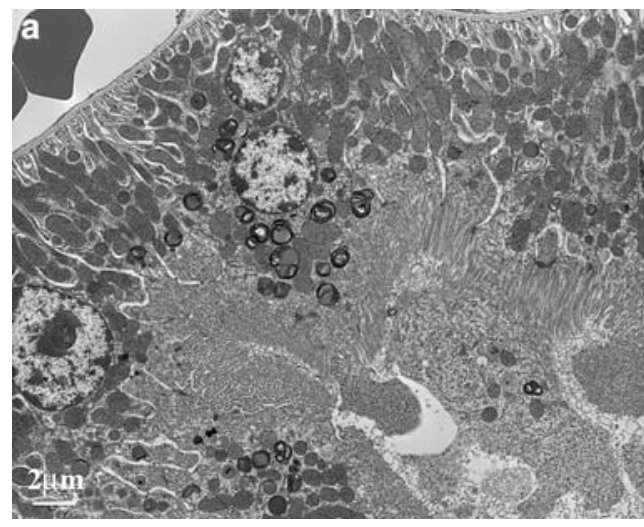

Fig. 4a,b Morphological effect of repeated agalsidase beta administration on renal tubular cells of Fabry mice. Three milligrams agalsidase beta/kg body weight was injected into Fabry mice every week for 4 weeks; the mice were killed 6 days after the last

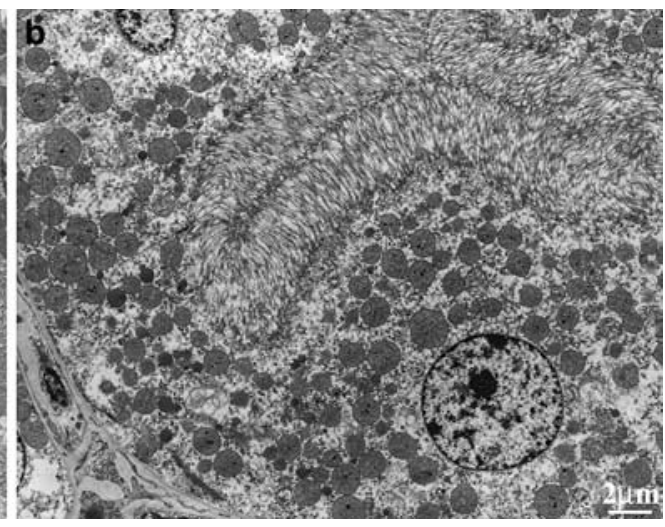

injection and kidney tissues were examined by electron microscopy. a Untreated - many lamellar inclusion bodies can be observed in the renal tubular cells. b Treated - the number of lamellar inclusion bodies is apparently decreased. Bars $2 \mu \mathrm{m}$ 
important. Fabry disease affects mainly the kidneys and heart, and most lysosomal matrix enzymes, including $\alpha$-galactosidase, are incorporated into these organs and cultured fibroblasts via M6P receptors on the plasma membrane (Kornfeld and Sly 2001). However, asialoglycoprotein receptors are involved in the uptake of these enzymes in the liver (Rosenfeld et al. 1986). Thus, successful targeting of $\alpha$-galactosidase in Fabry disease is strongly dependent on the presence of M6P residues on the sugar chains of the enzyme preparations. We examined the M6P residue content in our agalsidase alfa and agalsidase beta samples, and found that the M6P content in agalsidase beta is higher than that in agalsidase alfa. This result is in agreement with that reported by Lee et al. (2003).

We next examined the increase in $\alpha$-galactosidase activity in cultured human Fabry fibroblasts upon administration of agalsidase alfa and agalsidase beta. When the enzymes were added to the culture medium, the activity of cell-associated $\alpha$-galactosidase, which comprises that bound to cell membranes as well as that incorporated into cells, was apparently increased in both cases. However, the increase was always higher for agalsidase beta than for agalsidase alfa.

We also examined the increase in $\alpha$-galactosidase activity in organs of Fabry mice upon administration of agalsidase alfa and agalsidase beta. Considering previously reported analytical results obtained for Fabry mice (Lee et al. 2003; Ioannou et al. 2001), we chose 0.5 and $3.0 \mathrm{mg} / \mathrm{kg}$ body weight as the low and high doses for injection, respectively. We adjusted the amount of enzyme protein and administered the same amounts of enzymes to Fabry mice. A marked increase in $\alpha$-galactosidase activity was observed in all organs examined in both cases, but agalsidase beta apparently exhibited a higher enzyme activity increase in the kidneys, heart and spleen. The higher M6P content of agalsidase beta is thought to have been beneficial for uptake by these organs. As for the liver, an almost identical level of uptake of enzyme protein was observed in both cases. According to the results of monosaccharide analysis by Lee et al. (2003), the sialic acid/galactose ratio of agalsidase alfa $(0.56)$ is lower than that of agalsidase beta $(0.88)$, suggesting that agalsidase alfa contains more asialylated complex type sugar chains (Lee et al. 2003). This may explain why no difference was found between the agalsidase alfa and agalsidase beta samples as to the degree of enzyme activity increase in the liver, although the original enzyme activity in the agalsidase alfa sample obtained for this study was lower than that in the agalsidase beta sample.

The effect of agalsidase beta on degradation of accumulated GL-3 in cultured Fabry fibroblasts was examined. An immunocytochemical analysis revealed that the incorporated enzyme protein degraded GL-3 accumulated in cultured Fabry fibroblasts in a dosedependent manner, and that the effect was maintained for at least 7 days after the addition of agalsidase beta. Cleavage of accumulated GL-3 in renal tubular cells upon repeated injection of agalsidase beta was also observed morphologically. Thus, the effect of the recombinant $\alpha$-galactosidase was demonstrated.

In conclusion, we compared the biochemical characteristics and the effects of agalsidase alfa and agalsidase beta on cultured Fabry fibroblasts and Fabry mice. The administration of these drugs caused marked increases in $\alpha$-galactosidase activity in human Fabry cells and Fabry mouse tissues. However, the enzyme activity increases in cultured fibroblasts, kidneys, heart and spleen were higher for agalsidase beta than for agalsidase alfa. This may have resulted from differences in M6P residue content in the sugar chains of the two preparations. Agalsidase beta was incorporated into cultured Fabry fibroblasts and successfully cleaved accumulated GL-3 in the cells.

Acknowledgements We wish to thank Dr. Ashok B. Kulkarni (Gene Targeting Facility and Functional Genomics Unit, NIDCR, $\mathrm{NIH}$ ) for providing us with the Fabry mice, and also thank Sumitomo Pharmaceuticals for providing us with the agalsidase alfa sample. This work was partly supported by grants from the Tokyo Metropolitan Government, the Japan Society for the Promotion of Science, the Ministry of Education, Science, Sports and Culture, and the Ministry of Health, Labor and Welfare of Japan.

\section{References}

Desnick RJ, Ioannou YA, Eng CM (2001) $\alpha$-Galactosidase A deficiency: Fabry disease. In: Scriver CR, Beaudet AL, Sly WS, Valle D (eds) The metabolic and molecular bases of inherited disease, 8th edn. McGraw-Hill, New York, pp 37333774

Desnick RJ, Brady RO, Barranger J, Collins AJ, Germain DP, Goldman M, Grabowski G, Packman S, Wilcox WR (2003) Fabry disease, an under-recognized multisystemic disorder: expert recommendations for diagnosis, management, and enzyme replacement therapy. Ann Int Med 138:338-346

Eng CM, Banikazemi M, Gordon RE, Goldman M, Phelps R, Kim L, Gass A, Winston J, Dikman S, Fallon JT, Brodie S, Stacy CB, Mehta D, Parsons R, Norton K, O'Callaghan M, Desnick RJ (2001a) A phase $1 / 2$ clinical trial of enzyme replacement in Fabry disease: pharmacokinetic, substrate clearance, and safety studies. Am J Hum Genet 68:711-722

Eng CM, Guffon N, Wilcox WR, Germain DP, Lee P, Waldek S, Caplan L, Linthorst GE, Desnick RJ (2001b) Safety and efficacy of recombinant human $\alpha$-galactosidase A replacement therapy in Fabry's disease. N Engl J Med 345:9-16

Ioannou YA, Zeidner KM, Gordon RE, Desnick RJ (2001) Fabry disease: preclinical studies demonstrate the effectiveness of $\alpha$ galactosidase A replacement in enzyme-deficient mice. Am $\mathrm{J}$ Hum Genet 68:14-25

Ishii S, Kase R, Sakuraba H, Fujita S, Sugimoto M, Tomita K, Semba T, Suzuki Y (1994) Human $\alpha$-galactosidase gene expression: significance of two peptide regions encoded by exons 1-2 and 6. Biochim Biophys Acta 1204:265-270

Kornfeld S, Sly WS (2001) I-cell disease and pseudo-Hurler polydystrophy: disorders of lysosomal enzyme phosphorylation and localization. In: Scriver CR, Beaudet AL, Sly WS, Valle D (eds) The metabolic and molecular bases of inherited disease, 8th edn. McGraw-Hill, New York, pp 3469-3482

Kotani M, Kawashima I, Ozawa H, Ogura K, Ariga T, Tai T (1994) Generation of one set of murine monoclonal antibodies specific for globo-series glycolipids: evidence for differential distribution of the glycolipids in rat small intestine. Arch Biochem Biophys 310:89-96 
Lee K, Jin X, Zhang K, Copertino L, Andrews L, Baker-Malcolm J, Geagan L, Qiu H, Seiger K, Barngrover D, McPherson JM, Edmunds T (2003) A biochemical and pharmacological comparison of enzyme replacement therapies for the glycolipid storage disorder Fabry disease. Glycobiology 13:305-313

Mayes JS, Scheerer JB, Sifers RN, Donaldson ML (1981) Differential assay for lysosomal $\alpha$-galactosidase in human tissues and its application to Fabry's disease. Clin Chim Acta 112:247-251

Mehta A, Ricci R, Widmer U, Dehout F, Garcia de Lorenzo A, Kampmann C, Linhart A, Sunder-Plassmann G, Ries M, Beck M (2004) Fabry disease defined: baseline clinical manifestations of 366 patients in the Fabry Outcome Survey. Eur J Clin Invest 34:236-242

Nakao S, Takenaka T, Maeda M, Kodama C, Tanaka A, Tahara M, Yoshida A, Kuriyama M, Hayashibe H, Sakuraba H, Tanaka H (1995) An atypical variant of Fabry's disease in men with left ventricular hypertrophy. N Engl J Med 333:288-293

Ohshima T, Murray GJ, Swaim WD, Longenecker G, Quirk JM, Cardarelli CO, Sugimoto Y, Pastan I, Gottesman MM, Brady RO, Kulkarni AB (1997) $\alpha$-Galactosidase A deficient mice: a model of Fabry disease. Proc Natl Acad Sci USA 94:2540-2544

Ohshima T, Schiffmann R, Murray GJ, Kopp J, Quirk JM, Stahl S, Chan C-C, Zerfas P, Tao-Cheng J-H, Ward JM, Brady RO, Kalkarni AB (1999) Aging accentuates and bone marrow transplantation ameliorates metabolic defects in Fabry disease mice. Proc Natl Acad Sci USA 96:6423-6427
Rosenfeld EL, Belenky DM, Bystrov NK (1986) Interaction of hepatic asialoglycoprotein receptor with asialoorosomucoid and galactolyzed lysosomal $\alpha$-glucosidase. Biochim Biophys Acta 883:306-312

Schiffmann R, Murray GJ, Treco D, Daniel P, Sellos-Moura M, Myers M, Quirk JM, Zirzow GC, Borowski M, Loveday K, Anderson T, Gillespie F, Cliver KL, Jeffries NO, Doo E, Liang TJ, Kreps C, Gunter K, Frei K, Crutchfield K, Selden RF, Brady RO (2000) Infusion of $\alpha$-galactosidase A reduces tissue globotriaosylceramide storage in patients with Fabry disease. Proc Natl Acad Sci USA 97:365-370

Takashiba M, Chiba Y, Arai E, Jigami Y (2004) Analysis of mannose-6-phosphate labeled with 8-aminopyrene-1,3,6-trisulfonate by capillary electrophoresis. Anal Biochem 332:196198

Thurnberg BL, Rennke H, Colvin RB, Dikman S, Gordon RE, Collins AB Desnick RJ, O'Callaghan M (2002) Globotriaosylceramide accumulation in the Fabry kidney is cleared from multiple cell types after enzyme replacement therapy. Kidney Int 62:1933-1946 